\section{Case Reports in Nephrology and Dialysis}

Case Rep Nephrol Dial 2021;11:55-62

DOI: $10.1159 / 000512229$

Published online: February 5, 2021

(C) 2021 The Author(s)

Published by S. Karger AG, Basel www.karger.com/cnd

This article is licensed under the Creative Commons Attribution-NonCommercial 4.0 International License (CC BY-NC) (http://www.karger.com/Services/OpenAccessLicense). Usage and distribution for commercial purposes requires written permission.

\title{
Late-Onset Kidney Biopsy- Associated Retroperitoneal Hemorrhage in Lupus Nephritis: A Case Report of Pseudoaneurysm and Microaneurysm Formations
}

\author{
Panupong Hansrivijit Kinjal P. Gadhiya Sandra D. Zelonis \\ John T. Cinicola \\ Department of Internal Medicine, University of Pittsburgh Medical Center Pinnacle, \\ Harrisburg, PA, USA
}

\section{Keywords}

Renal artery $\cdot$ Pseudoaneurysm $\cdot$ Retroperitoneal hemorrhage $\cdot$ Renal hematoma $\cdot$ Kidney biopsy

\begin{abstract}
Late-onset retroperitoneal hemorrhage from renal intraparenchymal pseudoaneurysm (RIP) following a kidney biopsy is an extremely rare complication but should not be ignored, especially in high-risk populations. Here, we introduce a 32-year-old Caucasian female who presented with sudden-onset left-sided flank pain. She had recently been diagnosed with systemic lupus erythematosus (SLE) and had undergone a computed tomography (CT)-guided core needle biopsy of the left kidney 9 days earlier. The results were consistent with lupus nephritis class III or IV. Initial vitals were within normal limits. She appeared pale and her left flank was tender to palpation without discoloration or abdominal distention. Laboratory investigations showed a hemoglobin level of $7.1 \mathrm{~g} / \mathrm{dL}$. The CT scan of the abdomen and pelvis revealed a large hyperdense left perinephric collection consistent with perinephric hematoma with a moderate amount of retroperitoneal stranding most prominent on the left side extending across the midline to the right side. Contrast extravasation was suspected in the lower pole of
\end{abstract}




\section{Case Reports in Nephrology and Dialysis}

Hansrivijit et al.: Late-Onset Kidney Biopsy-Associated Retroperitoneal Hemorrhage in a Lupus Nephritis Patient

the left kidney consistent with active bleeding site. Emergent renal angiography revealed a $2 \times 1 \mathrm{~cm}$ intraparenchymal pseudoaneurysm in the lower pole of the left kidney along with a few small microaneurysms. Coil embolization of the pseudoaneurysm was successfully performed without any complications. In conclusion, SLE or lupus nephritis in this patient may be the predisposing factors for microaneurysm and RIP formations. RIP is an unusual complication after percutaneous kidney biopsy that carries a significant mortality rate if ruptured, causing retroperitoneal hemorrhage. Clinicians should be vigilant when encountering high-risk patients with persistent hematuria, flank pain, or abdominal pain within four weeks after a kidney biopsy.

(C) 2021 The Author(s)

Published by S. Karger AG, Basel

\section{Background}

Percutaneous kidney biopsy is the gold standard procedure aiding in the diagnosis and treatment of several kidney diseases. Kidney biopsy is generally safe and carries minimal morbidity and mortality [1]. Since its first introduction in 1951 by Iversen and Brun, the biopsy techniques and incorporation of advances in radiographic modality have led to significant improvement in minimizing immediate complications from kidney biopsy [1].

Immediate ( $<24 \mathrm{~h}$ post-procedure) minor complications following a kidney biopsy are common. These include gross hematuria (3.5\%) and hematoma formation visible on computed tomography (CT) scan (57-91\%). These complications are minor and do not require invasive intervention. The risks for major complications, such as need for blood transfusion, nephrectomy, bladder obstruction, or death, are considerably low, with an incidence of less than $1 \%[1]$.

Late-onset retroperitoneal hemorrhage following a kidney biopsy is an extremely rare complication, yet should not be ignored. Lees et al. [2] retrospectively reviewed 2,563 patients with nephrologist-performed kidney biopsy and discovered that late-onset retroperitoneal hemorrhage occurred only in 1 patient $(0.04 \%)$. Unfortunately, this patient did not survive. In this study, patients with vasculitis had the highest incidence of major bleeding after a percutaneous kidney biopsy. Here, we present a case of late-onset retroperitoneal hemorrhage due to renal artery pseudoaneurysm 9 days after percutaneous kidney biopsy in a patient with untreated lupus nephritis who was found to have preexisting microaneurysms.

\section{Case Presentation}

A 32-year-old Caucasian female with a history of recently diagnosed systemic lupus erythematosus (SLE) presented to the emergency department with sudden-onset left-sided flank pain. The pain was dull and constant in origin with a score of 10 out of 10 . She reported nausea but denied dysuria, hematuria, fever, chills, chest pain, shortness of breath, or changes in bowel function.

Seven weeks prior to this admission, she was hospitalized for autoimmune hemolytic anemia with a nadir hemoglobin level of $4.6 \mathrm{~g} / \mathrm{dL}$. Her hematologic workups were remarkable for positive antiglobulin test (Coombs test), nuclear dense fine speckled pattern on antinuclear antibodies screening with 1:640 titer, and positive anti-double-stranded DNA antibody of $259 \mathrm{IU} / \mathrm{mL}$. Her recent C3 and C4 levels were diminished at 27 and $4 \mathrm{mg} / \mathrm{dL}$, respectively. She

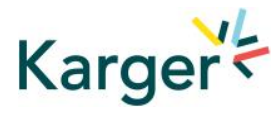




\section{Case Reports in Nephrology and Dialysis}

Hansrivijit et al.: Late-Onset Kidney Biopsy-Associated Retroperitoneal Hemorrhage in a Lupus Nephritis Patient

was treated with intravenous methylprednisolone followed by packed red cell transfusion. Her hospital course was complicated by acute kidney injury with a presenting serum creatinine level of $2.20 \mathrm{mg} / \mathrm{dL}$. Urine analysis showed 3+ occult blood and 2+ protein with a urine protein-creatinine ratio of $1.95 \mathrm{~g} / \mathrm{g}$. CT of the abdomen and pelvis was notable for small pericardial effusion and normal renal morphology. She was started on intravenous fluid and her serum creatinine level gradually improved. Her anemia and acute kidney injury improved, thus she was discharged home. At that time her hemoglobin level was $8.9 \mathrm{~g} / \mathrm{dL}$ and her serum creatinine level was $1.22 \mathrm{mg} / \mathrm{dL}$. Kidney biopsy was arranged as an outpatient procedure.

One week after discharge (or 9 days prior to the recent admission), she underwent a CTguided core needle biopsy of the left kidney. The cortex was obtained from the lower pole of the left kidney. There were no immediate complications and the patient was discharged on the same day. The kidney biopsy showed immune complex-mediated proliferative glomerulonephritis, 2/5 global glomerulosclerosis, and mild arteriolar hyalinosis. These findings were consistent with International Society of Nephrology/Renal Pathology Society class III or IV lupus nephritis. No structures similar to small arterial aneurysmal wall were identified from the obtained core tissue. Induction therapy was not initiated at that time.

In this recent admission, the patient presented with sudden-onset left-sided flank pain. Initial vital signs were a temperature of $36.4^{\circ} \mathrm{C}$, a heart rate of $100 / \mathrm{min}$, a respiratory rate of $20 / \mathrm{min}$, a blood pressure of $151 / 108 \mathrm{~mm} \mathrm{Hg}$, and an oxygen saturation of $100 \%$ on room air. She appeared pale and her left flank was tender to palpation, without discoloration or abdominal distention. Other parts of physical examination were unremarkable.

\section{Investigations}

Initial investigations demonstrated hemoglobin $7.1 \mathrm{~g} / \mathrm{dL}$, hematocrit $20.5 \%$, platelets $183,000 / \mu \mathrm{L}$, and international normalization ratio 1.1. Metabolic panel showed serum sodium $140 \mathrm{mEq} / \mathrm{L}$, serum potassium $3.8 \mathrm{mEq} / \mathrm{L}$, serum chloride $104 \mathrm{mEq} / \mathrm{L}$, serum bicarbonate $28.1 \mathrm{mEq} / \mathrm{L}$, serum creatinine $1.11 \mathrm{mg} / \mathrm{dL}$, and blood urea nitrogen $24 \mathrm{mg} / \mathrm{dL}$.

A CT scan of the abdomen and pelvis with intravenous contrast medium (Fig. 1) revealed a large hyperdense left perinephric collection consistent with perinephric hematoma with a moderate amount of retroperitoneal stranding most prominent on the left side extending across the midline to the right side. The retroperitoneal stranding stems from the level of the left hemidiaphragm to the left lower pelvis. Contrast extravasation was suspected in the lower pole of the left kidney consistent with active bleeding site. Chronic stranding in the right perinephric space was also noted.

\section{Treatment}

The patient underwent emergent selective left renal artery coil embolization. The procedure was successful without any complications. Left renal angiography showed an approximately $2 \times 1 \mathrm{~cm}$ intraparenchymal pseudoaneurysm in the lower pole of the left kidney with active extravasation. The coils were placed in the medial and lateral branches supplying the pseudoaneurysm (Fig. 2). Small microaneurysms were also noted on angiography.

\section{Clinical Follow-Up}

The patient followed with her nephrologist and hematologist after hospital discharge. Her flank pain completely resolved, and she received an induction therapy followed by maintenance therapy for her lupus nephritis.

\section{Karger'=}




\section{Case Reports in Nephrology and Dialysis}

Case Rep Nephrol Dial 2021;11:55-62

DOI: 10.1159/000512229

c) 2021 The Author(s). Published by S. Karger AG, Basel www.karger.com/cnd

Hansrivijit et al.: Late-Onset Kidney Biopsy-Associated Retroperitoneal Hemorrhage in a Lupus Nephritis Patient

\section{Discussion}

Here, we present a patient with newly diagnosed, untreated lupus nephritis who presented with late-onset retroperitoneal hemorrhage 9 days after a percutaneous native kidney biopsy. A renal intraparenchymal pseudoaneurysm (RIP) and some microaneurysms were identified from renal angiography.

A large number of native kidney diseases have been shown to be associated with renal hemorrhage. If renal hemorrhage occurs spontaneously (nontraumatic), it is known as Wunderlich syndrome, named after Carl Wunderlich who described it in 1856 [3]. Patients may present with the classic Lenk triad: acute flank pain or abdominal pain, palpable mass, and hypovolemia [4]. Malignancies, cystic renal diseases, vasculitis, infectious processes, and aneurysms/pseudoaneurysms are potential causes of renal hemorrhage [4]. Among all causes of vasculitis, polyarteritis nodosa is the most common cause of Wunderlich syndrome (>80\%), followed by SLE-related renal artery microaneurysms [5]. This finding also corresponds with Lees et al. [2], who showed that patients with vasculitis had the highest incidence of major bleeding after a percutaneous kidney biopsy. In this patient, renal angiography incidentally identified some microaneurysm in the left kidney vasculature. Given positive anti-doublestranded DNA antibody, negative antineutrophil cytoplasmic antibody tests, and low complement levels, we are convinced that the microaneurysms in this patient are most likely associated with SLE or lupus nephritis, which are potential risk factors for renal hemorrhage.

The formation and growth of microaneurysms is influenced by endothelial changes following hemodynamic changes. A fluctuation in local blood pressure creates wall sheer stress which results in endothelial remodeling and subsequently changes in aneurysmal geometry $[6,7]$. Thus, it is possible that immune complex-mediated endothelial injury from renal vasculitis led to aneurysm formation in our patient.

Unlike true aneurysm, in which luminal dilatation is encapsulated by the natural arterial walls, the pseudoaneurysm walls derive from the surrounding tissues adjacent to the injury site [8]. These tissues are formed by degradation of blood clots and necrotic tissues, resulting in recanalization of the blood vessels which sequentially leads to pseudoaneurysm [8]. However, the integrity of this wall is fragile and prone to rupture when normal blood flow is restored [9]. Although the causal relationship between SLE/lupus nephritis and pseudoaneurysm formation has not been fully established in the literature, giant dorsalis pedis pseudoaneurysm following arterial line cannulation [10], and pancreatic pseudoaneurysms from SLE-induced pancreatitis [11] were described in SLE patients. From our speculation, it is possible that the inflammation induced by the trauma from core needle kidney biopsy would be exaggerated in patients with SLE or lupus nephritis, leading to the formation of pseudoaneurysms.

RIP is a very uncommon but life-threatening condition. RIP after a percutaneous kidney biopsy is rare and often described in case reports [1,12-15]. One series of 72 percutaneous allograft biopsies estimated the incidence of RIP to be approximately 5\% [16]. The majority of RIPs in this study spontaneously resolved without causing any significant bleeding [16]. Tøndel et al. [17] reported that the incidence of surgical/arterial embolization after percutaneous kidney biopsy was only $0.2 \%$. However, the complications in this cohort was immediate and less likely due to RIP formation. Thus, given the evidence to date, one could say that retroperitoneal hemorrhage from RIP is extremely rare.

Most cases of RIP are asymptomatic. However, presenting symptoms can vary from abdominal tenderness, flank pain, and hematuria to hypovolemic shock or even hypertension [1,

\section{Karger'=}




\section{Case Reports in Nephrology and Dialysis}

Hansrivijit et al.: Late-Onset Kidney Biopsy-Associated Retroperitoneal Hemorrhage in a Lupus Nephritis Patient

12-15]. Hematuria is the most common presenting symptom of RIP [18]. The onset of symptomatic RIP is usually late, ranging from 1 to 4 weeks after injury. One study showed that the average duration from renal injury to symptom onset was 17 days [2, 9]. Persistent hematuria and flank pain 1 week after percutaneous kidney biopsy should warrant clinicians to consider complicated RIP in their differential diagnosis as patient outcomes are dependent on the time from diagnosis to treatment.

Diagnosing RIP is challenging. Renal angiography has been considered the standard tool for diagnosis. However, CT angiography, color Doppler sonography, or magnetic resonance angiography also have satisfactory diagnostic yield [19]. In CT angiography, the RIP is best visualized on the arterial phase, and sometimes on the nephrographic phase. During the pyelographic phase, the contrast medium is excreted and thus RIP cannot be visualized [19]. For ultrasonographic imaging, RIPs may resemble a cystic lesion on plain ultrasound, but show a characteristic to-and-fro flow within the mass on color Doppler [19]. Renal angiography is recommended to evaluate RIP especially if the imaging findings are inconclusive or clinicians have a high index of suspicion.

Currently, there is no existing consensus regarding the RIP size that requires intervention. Although the risk of RIP rupture is perceivably low, it is associated with a mortality rate of up to $80 \%$ [20]. Most experts suggest that aneurysms $>2 \mathrm{~cm}$ in diameter have a high risk for rupture. Whether the growth of aneurysms should be considered as part of treatment planning is unknown. Angiographic embolization, open vascular surgery, or nephrectomy are possible treatment options [18]. In general, angiographic embolization is usually the treatment of choice due to its selectivity to preserve renal parenchyma and its minimally invasive nature [21].

\section{Conclusions}

SLE or lupus nephritis in this patient may be the predisposing factors for microaneurysm and RIP formations. Moreover, RIP is an unusual complication after percutaneous kidney biopsy that carries a significant mortality rate if ruptured, causing retroperitoneal hemorrhage. Clinicians should be vigilant when encountering high-risk patients with persistent hematuria, flank pain, or abdominal pain within 4 weeks after a kidney biopsy.

\section{Acknowledgments}

We would like to thank the Department if Interventional Radiology of University of Pittsburgh Medical Center Pinnacle Hospitals for taking care of this patient.

\section{Statement of Ethics}

Written informed consent for publication including images was obtained from the reported patient. The research was conducted in accordance with the World Medical Association Declaration of Helsinki.

\section{Karger'=}




\section{Case Reports in Nephrology and Dialysis}

\section{Conflict of Interest Statement}

The authors declare no potential conflicts of interest.

\section{Funding Sources}

There is no funding associated with this article.

\section{Author Contributions}

P. Hansrivijit, K.P. Gadhiya, and S.D. Zelonis obtained the history, performed the physical examinations, and obtained investigation reports. P. Hansrivijit edited the manuscript prior to submission. P. Hansrivijit and J.T. Cinicola revised the manuscript prior to publication.

\section{References}

1 Mallat SG, Abou Arkoub R, El Achkar B, Saade C, El-Merhi F. Renal pseudoaneurysm formation post allograft biopsy: a case report. BJR Case Rep. 2016 Dec;3(2):20150502.

2 Lees JS, McQuarrie EP, Mordi N, Geddes CC, Fox JG, Mackinnon B. Risk factors for bleeding complications after nephrologist-performed native renal biopsy. Clin Kidney J. 2017 Aug;10(4):573-7.

3 Wunderlich CA. Handbuch der Pathologie und Therapie. Stuttgart: Ebner \& Seubert; 1976.

4 Chao CT, Wang WJ, Ting JT. Wünderlich syndrome from lupus-associated vasculitis. Am J Kidney Dis. 2013 Jan;61(1):167-70.

5 Brkovic D, Moehring K, Doersam J, Pomer S, Kaelble T, Riedasch G, et al. Aetiology, diagnosis and management of spontaneous perirenal haematomas. Eur Urol. 1996;29(3):302-7.

6 Mimori A, Suzuki T, Hashimoto M, Nara H, Yoshio T, Masuyama JI, et al. Subarachnoid hemorrhage and systemic lupus erythematosus. Lupus. 2000;9(7):521-6.

7 Yasuhara T, Tamiya T, Sugiu K, Inoue S, Ohmoto T. De novo formation and rupture of an aneurysm. Case report. J Neurosurg. 2002 Sep;97(3):697-700.

8 Srivastava A, Singh KJ, Suri A, Dubey D, Kumar A, Kapoor R, et al. Vascular complications after percutaneous nephrolithotomy: are there any predictive factors? Urology. 2005 Jul;66(1):38-40.

9 Lee RS, Porter JR. Traumatic renal artery pseudoaneurysm: diagnosis and management techniques. J Trauma. 2003 Nov;55(5):972-8.

10 Anastasiadou C, Giannakakis S, Galyfos G, Livieratos L, Kastrisios G, Papapetrou A, et al. Giant Dorsalis Pedis Pseudoaneurysm Following Cannulation for Arterial Line in a Patient with Systemic Lupus Erythematosus. Vasc Spec Int. 2019 Jun;35(2):114-7.

11 Hoorn EJ, Flink HJ, Kuipers EJ, Poley JW, Mensink PB, Dolhain RJ. Complicated systemic lupus erythematosus pancreatitis: pseudocyst, pseudoaneurysm, but real bleeding. Lupus. 2011 Mar;20(3):305-7.

12 Rivera M, Villacorta J, Jiménez-Alvaro S, Quereda C. Asymptomatic large extracapsular renal pseudoaneurysm following kidney transplant biopsy. Am J Kidney Dis. 2011 Jan;57(1):175-8

13 Yang HK, Koh ES, Shin SJ, Chung S. Incidental renal artery pseudoaneurysm after percutaneous native renal biopsy. BMJ Case Rep. 2013 Feb;2013:bcr2012006537.

14 Yun GY, Kim SK, Park SK, Moon SJ, Lee JE, Song SW, et al. Asymptomatic renal pseudoaneurysm after percutaneous renal biopsy. Kidney Res Clin Pract. 2013 Jun;32(2):87-9.

15 Antonopoulos IM, Yamacake KG, Tiseo BC, Carnevale FC, Junior EZ, Nahas WC. Renal pseudoaneurysm after core-needle biopsy of renal allograft successfully managed with superselective embolization. Int Braz J Urol. 2016 Jan-Feb;42(1):165-7.

16 Brandenburg VM, Frank RD, Riehl J. Color-coded duplex sonography study of arteriovenous fistulae and pseudoaneurysms complicating percutaneous renal allograft biopsy. Clin Nephrol. 2002 Dec;58(6):398-404.

17 Tøndel C, Vikse BE, Bostad L, Svarstad E. Safety and complications of percutaneous kidney biopsies in 715 children and 8573 adults in Norway 1988-2010. Clin J Am Soc Nephrol. 2012 Oct;7(10):1591-7.

18 Albani JM, Novick AC. Renal artery pseudoaneurysm after partial nephrectomy: three case reports and a literature review. Urology. 2003 Aug;62(2):227-31. 


\section{Case Reports in Nephrology and Dialysis}

\begin{tabular}{l|l}
\hline Case Rep Nephrol Dial 2021;11:55-62 \\
\hline DOI: 10.1159/000512229 & $\begin{array}{l}\text { @ 2021 The Author(s). Published by S. Karger AG, Basel } \\
\text { www.karger.com/cnd }\end{array}$ \\
\hline
\end{tabular}

Hansrivijit et al.: Late-Onset Kidney Biopsy-Associated Retroperitoneal Hemorrhage in a Lupus Nephritis Patient

19 Helck A, Hoffmann RT, Sommer WH, Notohamiprodjo M, Albyaa H, Reiser M, et al. Diagnosis, therapy monitoring and follow up of renal artery pseudoaneurysm with contrast-enhanced ultrasound in three cases. Clin Hemorheol Microcirc. 2010;46(2-3):127-37.

20 Breyer BN, McAninch JW, Elliott SP, Master VA. Minimally invasive endovascular techniques to treat acute renal hemorrhage. J Urol. 2008 Jun;179(6):2248-52; discussion 2253.

21 Mima A, Toma M, Matsubara T, Shiota F, Iehara N, Abe H, et al. Angio-embolization of renal artery pseudoaneurysm after renal biopsy: a case report. Ren Fail. 2009;31(8):753-5.

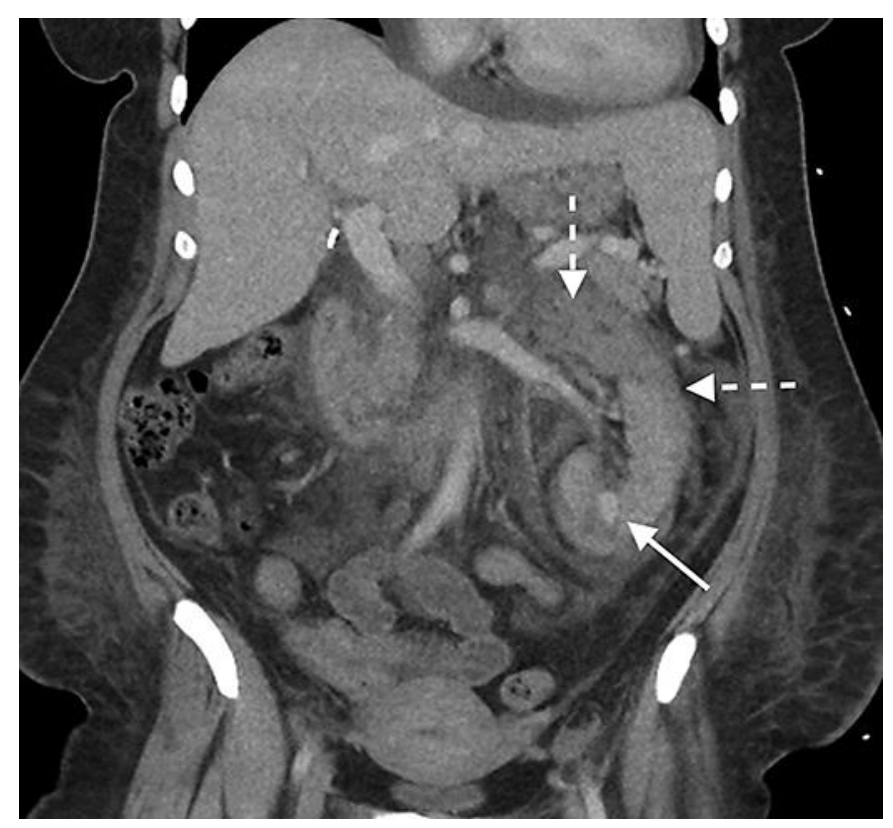

Fig. 1. Computed tomography scan with contrast medium. Hyperdense lesion at the left renal pole suggesting extravasation of the contrast medium (solid arrow) and large hyperdense left perinephric collection consistent with perinephric hematoma with moderate amount of retroperitoneal stranding (dashed arrows). 


\section{Case Reports in Nephrology and Dialysis}

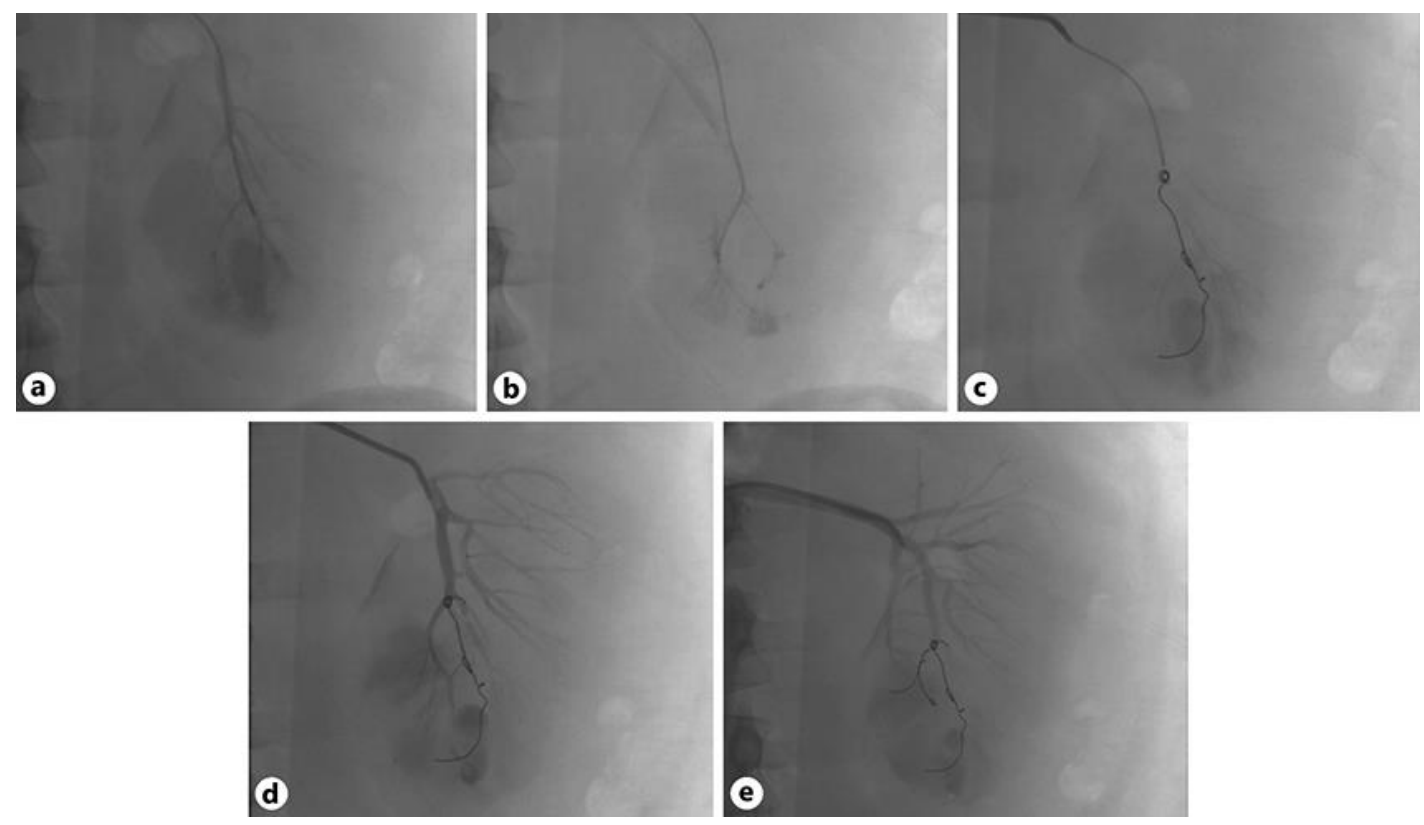

Fig. 2. Left renal angiography. a $2 \times 1 \mathrm{~cm}$ pseudoaneurysm at the left renal pole. $\mathbf{b}$ Small microaneurysms were noted after contrast medium injection into a different arterial branch. c Coil embolization of the artery (lateral branch) supplying the pseudoaneurysm. $\mathbf{d}$ Another arterial supply (medial branch) into the pseudoaneurysm was identified. e Successful coil embolization of another artery supplying the pseudoaneurysm. 